\title{
EVOLUÇÃO DA FERTILIDADE DE SOLOS CONSTRUÍDOS EM ÁREAS DE PASSIVO AMBIENTAL DA MINERAÇÃO DE CARVÃO, LAURO MULLER - SC
}

\section{EVOLUTION OF FERTILITY IN SOILS CONSTRUCTED IN AREAS IMPACTED BY COAL MINING, LAURO MULLER - SC}

\begin{abstract}
Daniel Pazini Pezente
Pesquisador Centro Tecnológico SATC. Aluno do programa de mestrado PPGCA - UNESC. Email:

daniel.pezente@satc.edu.br
\end{abstract}

William de Oliveira Sant Ana

Pesquisador Centro Tecnológico SATC. Aluno do programa de doutorado PPGCA - UNESC. Email:

william.santana@satc.edu.br

\section{RESUMO}

No município de Lauro Müller, durante mais de 50 anos operou a Companhia Nacional de Mineração de Carvão do Barro Branco, que também minerou pelo método de lavra a céu aberto, remanescendo pequenas áreas esparsas espacialmente, e impactadas ambientalmente. Num contexto recente, a Carbonífera Catarinense Ltda., por ter assumido esse passivo ambiental, efetuou a recuperação através da conformação topográfica, construção de solo e drenagens e estabelecimento de vegetação nessas áreas. Desde então, elas passaram a ser monitoradas ambientalmente pelo Núcleo de Meio Ambiente do Centro Tecnológico da Associação da Indústria Carbonífera de Santa Catarina, com vistas ao acompanhamento da evolução dos indicadores de qualidade ambiental. Com base no trabalho de monitoramento, selecionaram-se 6 campanhas amostrais de 12 parâmetros físico-químicos do solo de 6 distintas áreas (Locks, 3A3B, Bett, Rio do Meio, Zona 4 e Coreia II), cujos resultados objetivam analisar a evolução da fertilidade e capacidade de suporte desse substrato ao longo das campanhas, bem como comparar a velocidade de recuperação entre as áreas. As áreas foram percorridas em toda a sua extensão, realizando coletas avulsas de solos, a fim de gerar uma malha amostral homogênea e representativa. As amostras de cada área foram reunidas em uma amostra composta, homogeneizadas e quarteadas. Para se atingir os objetivos propostos, como materiais e métodos, os 12 parâmetros definidos foram de: percentual de argila, SMP, pH em água, fósforo, potássio, matéria orgânica, cálcio, magnésio, saturação por bases, capacidade de troca de cátions, e saturação por alumínio. A hipótese inicial é de que áreas geograficamente próximas, submetidas ao mesmo clima, de relevo similar e técnicas de recuperação idênticas, apresentem ritmos sincronizados na evolução da qualidade do substrato. Como tratamento estatístico, para que o correto relacionamento entre variáveis e destas com os casos, seja devidamente interpretado, se faz necessário normalizar os resultados analíticos, padronizá-los, para a aplicação da estatística multivariada. A técnica estatística adotada, a Análise de Componentes Principais (ACP), objetivou sintetizar as informações dos conjuntos das variáveis, ordenando os casos em relação aos parâmetros descritores, utilizando-se do software estatístico Multi-Variate Statistical Package $^{\circledR}$, na qual foi elaborado gráfico euclidiano bidimensional. Os resultados preliminares indicam que a maioria das áreas apresentou progressão positiva da qualidade do substrato, apesar da baixa estabilidade edáfica. As áreas Coreia II e Locks não demonstraram evolução edáfica para uma condição eutrófica. Nesse caso, recomendam-se estudos complementares para a tomada de decisão sobre possíveis reintervenções nestas áreas em específico.

Palavras-chave: Recuperação ambiental. Solos. Mineração. Passivos ambientais. 


\begin{abstract}
Within the municipality of Lauro Müller, in the District of Guatá, for more than 50 years, the National Coal Mining Company of Barro Branco operated, which also mined by the open-sky method, remaining small areas spatially sparse and impacted by the environment. In a recent context, Carbonífera Catarinense Ltda. carried out the environmental recovery through topographic conformation, soil construction and drainage and establishment of vegetation in these areas. Since then they have been monitored environmentally, in order to monitor the evolution of their indicators, by the Environment Center of the Technological Center of the Association of the Carboniferous Industry of Santa Catarina. From this monitoring work, we selected 8 sampling campaigns of 12 physicalchemical soil parameters from 6 different areas (4, 5, 13, Juliana, 16 and 20 ), whose results aim to analyze the evolution Fertility and ability to support this substrate throughout the campaigns, as well as to compare the recovery speed between areas. The areas were traversed in all their extension, making separate collections of soils, in order to generate a homogeneous and representative sample mesh. Samples from each area were collected in a composite sample, homogenized and quarteted. In order to reach the proposed objectives, as materials and methods, the 12 parameters defined were: Clay percentage, SMP, $\mathrm{pH}$ in water, Phosphorus, Potassium, Organic matter, Calcium, Magnesium, Base saturation, Cation exchange capacity, And Saturation by aluminum. The initial hypothesis is that geographically close areas, subject to the same climate, of similar relief and identical recovery techniques, present synchronized rhythms in the evolution of substrate quality. As a statistical treatment, it is necessary to standardize the analytical results, standardize them, for the application of multivariate statistics, so that the correct relationship between variables and these variables with the cases is properly interpreted. The statistical technique adopted, the Principal Component Analysis (PCA), aimed to synthesize the information of the sets of variables, ordering the cases in relation to the descriptor parameters, using the statistical software MultiVariate Statistical Package ${ }^{\circledR}$, in which a graph Two-dimensional Euclidean. Preliminary results indicate that most areas showed a positive progression of substrate quality, despite the low edaphic stability. The Coreia II and Locks areas did not show edaphic evolution to a eutrophic condition. In this case, complementary studies are recommended to make a decision about possible reinterventions in these specific areas.
\end{abstract}

Keywords: Environmental recovery. Soil. Mining. Environmental liabilities. 


\section{INTRODUÇÃO}

A atividade de mineração de carvão é parte fundamental da história do sul catarinense, sendo que, para alguns municípios, constitui-se na essência da sua própria história, como Criciúma, Siderópolis e Lauro Müller (BELLOLI et al., 2002). Apesar da sua importância econômica, a mineração de carvão, se executada sem critérios técnicos e legais, causa sérios problemas socioambientais, em quaisquer de suas etapas do processo produtivo, podendo ocorrer a liberação de poluentes, impactando negativamente a paisagem, as comunidades faunísticas e florísticas, bem como acarretando na contaminação do solo, do ar e dos recursos hídricos (TORREZANI; OLIVEIRA, 2013).

A principal fonte de degradação ambiental gerada por essa atividade está relacionada aos rejeitos do beneficiamento do carvão, ricos em dissulfeto de ferro $\left(\mathrm{FeS}_{2}\right)$, conhecido como pirita. Esse elemento entra em processo de oxidação quando em contato com o ar e a água, e pela atividade de algumas bactérias, tais como Thibacilus ferroxidans, gera a Drenagem Ácida de Mina (DAM), comumente associada à dissolução de metais em minas abandonadas (FUNGARO; IZODORO 2006).

No estado de Santa Catarina, a mineração de carvão acontece em minas subterrâneas e a céu aberto, sendo que, no processo de mineração a céu aberto, a remoção das camadas superiores foi executada, em décadas passadas, de forma desordenada, provocando a inversão do solo, gerando pilhas de materiais geológicos que têm na sua base a camada fértil do solo, causando a perda de solo e a sua degradação (ARAÚJO et al., 2005).

De acordo com a resolução 001/86 do CONAMA (Conselho Nacional de Meio Ambiente), que institui a obrigatoriedade da apresentação de EIA/RIMA (Estudo de Impacto Ambiental/Relatório de Impacto Ambiental) para licenciamento de empreendimentos de mineração, o plano de lavra e o de reabilitação da área a ser minerada devem constar no projeto de mineração. A obrigatoriedade de reabilitar as áreas degradadas encontra-se regulamentada pelo decreto número 97.632, de 10 abril de 1989.

Dentre as estratégias de recuperação de áreas impactadas pela mineração na bacia carbonífera catarinense, de acordo com Pinto (1997), implementam-se solos construídos, que devem proporcionar condições para o desenvolvimento da vegetação, permitindo a fixação do solo, evitando a atuação de processos erosivos que podem expor os rejeitos e/ou estéril de cobertura e que favoreçam a geração da DAM (FANNING; FANNING, 1989).

O uso de cobertura vegetal como medida mitigadora dos impactos ambientais é uma opção prática e econômica, embora apresente dificuldades de adaptação à composição física e 
química do solo degradado. Sendo assim, deve-se escolher espécies com capacidade de crescimento rápido para, dessa forma, proteger o solo, abrigar e alimentar a fauna, recompor a paisagem e reestabelecer o regime hídrico, criando condições para o estabelecimento de outras espécies mais exigentes (ALVES; SOUZA, 2008).

A evolução das condicionantes químicas de um solo construído, em áreas de passivo ambiental, é de grande importância, pois permite realizar uma avaliação da metodologia de recuperação utilizada, servindo de base para o conhecimento da evolução da qualidade dos processos de recuperação ambiental.

Considerando a importância da fertilidade do solo para a manutenção, distribuição e abundância de espécies vegetais (RODRIGUES et al., 2007), bem como a capacidade de ciclagem de nutrientes e a autossustentabilidade, associado ao baixo número de estudos relacionados a solos construídos sobre áreas de mineração de carvão, o objetivo deste estudo foi analisar a evolução das condicionantes edáficas químicas de solos construídos em áreas mineradas a céu aberto, em áreas de passivo ambiental da mineração de carvão, utilizando-se de ferramentais de matriz de similaridade e gráfico bidimensional, obtidos por técnica de estatística multivariada.

\section{ÁREA DE ESTUDO}

O estudo foi desenvolvido no município de Lauro Muller/SC, onde se localizam diversas áreas que já comportaram no passado exploração de carvão mineral a céu aberto, cuja responsabilidade de recuperação foi atribuída à Carbonífera Catarinense Ltda., sendo que muitas já tiveram suas obras de recuperação ambiental realizadas. Considerando as condições de relevo, características geológicas e processos de recuperação similares, foram escolhidas as áreas Locks, 3A3B, Bett, Rio do Meio, Zona 4 e Coreia II, conforme pode ser visto na Figura 1. 
Figura 1 - Localização das áreas de estudo no interior no município de Lauro Müller

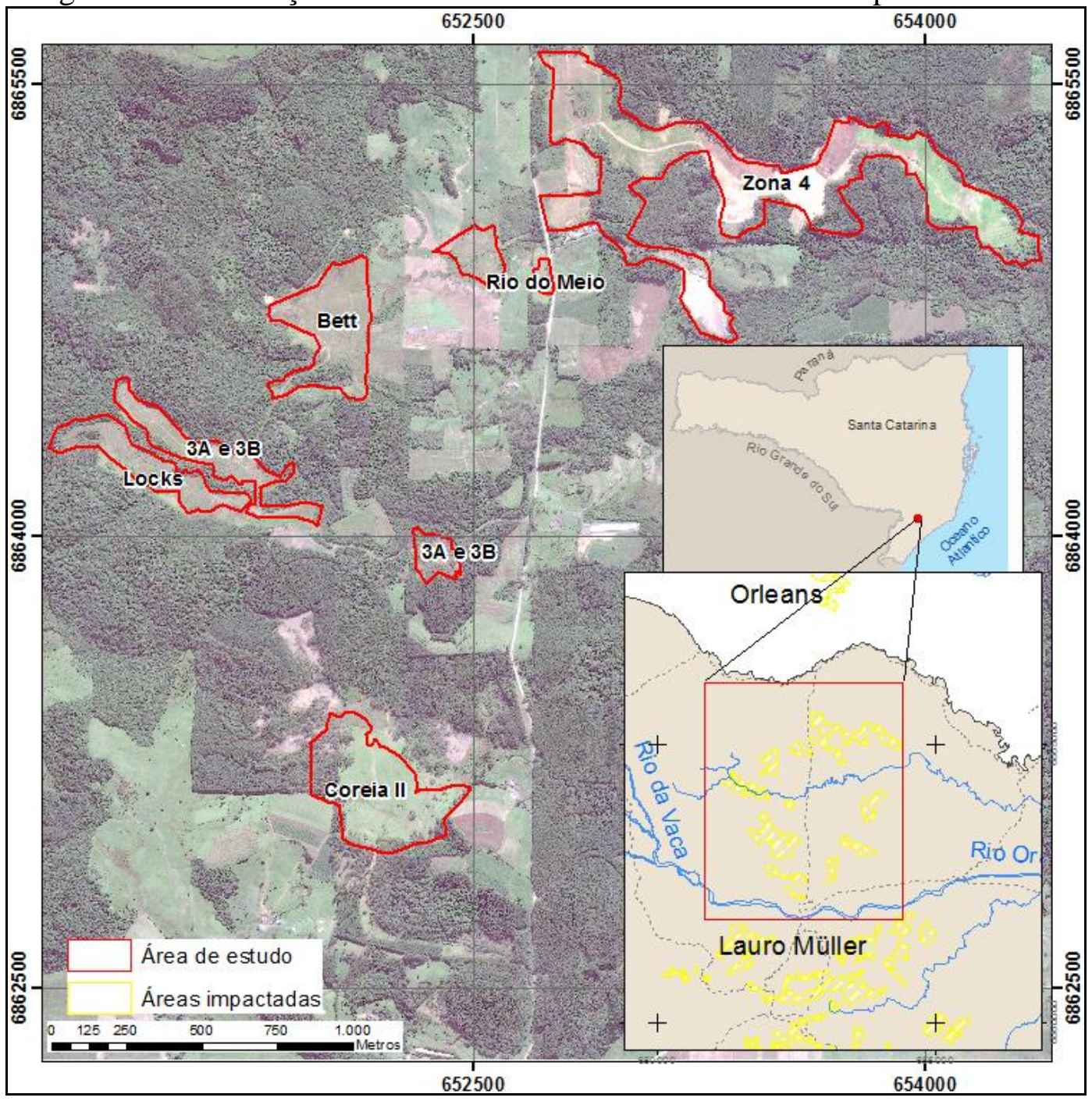

Fonte: Elaborada pelos autores.

A geologia local é constituída por rochas sedimentares das formações Gondwânicas da Bacia do Paraná, representadas localmente pelas formações Palermo e Rio Bonito, Grupo Guatá, especificamente a camada minerada Barro Branco (BRASIL, 1987). Campos et al. (2003) analisaram um solo próximo à área minerada e classificaram-no como Argissolo Vermelho-Amarelo (EMBRAPA, 2006).

De acordo com o sistema de classificação global dos tipos climáticos, proposto por Köppen-Geiger (PEEL; FINLAYSON; MC MAHON, 2007), a conjunção da atuação sazonal das diferentes massas de ar, acrescida de fatores como latitude, altitude e continentalidade, caracteriza a área de estudo como de clima temperado chuvoso, úmido em todas as estações, com verão quente, ou seja, do tipo Cfa, com temperaturas variando entre $17^{\circ}$ e $19,3^{\circ} \mathrm{C}$, precipitação pluviométrica média de $1.655 \mathrm{~mm}$ por ano (CIRAM, 2017) e com floresta original representada pela Floresta Ombrófila Densa (LEITE; KLEIN, 1990). 
Os trabalhos de mineração aconteceram de forma semelhante em todas as áreas, ou seja, segundo os mesmos critérios, seguindo o procedimento de conformação topográfica do terreno, recobrimento dos estéreis e rejeitos com uma camada de 50 centímetros de argila, sendo 20 centímetros de argila compactada e 30 centímetros de argila não compactada associada a corretivos e adubação para a formação do top soil. Após esse procedimento, foi inserida a vegetação, sendo usada a espécie herbácea Brachiaria sp., visto que essa tem características de crescimento rápido, podendo ser usada como forrageira e com boa adaptação às áreas de solo construído.

\section{METODOLOGIA}

\section{COLETA}

Nas áreas de passivo ambiental recuperada, foi percorrida toda a sua extensão, sendo realizadas coletas de subamostras de solo, no intuito de formar uma malha amostral homogênea e representativa, gerando uma amostra composta por área, seguindo a metodologia de amostragem de solo da Comissão de Química e Fertilidade do Solo - RS/SC (2016).

Essas subamostras foram transportadas até o laboratório de Geotecnia da Satc (LABGEO/SATC), onde foram homogeneizadas e quarteadas para a obtenção de uma amostra composta que representasse as reais condições da área amostrada.

As amostras compostas foram encaminhadas ao Laboratório de Análise de Solo da EPAGRI, na Estação Experimental de Ituporanga, sendo que, na $10^{\circ}$ campanha, as análises físico-químicas foram realizadas pelo laboratório de solos da UDESC. Os parâmetros físicos e químicos analisados compõem a análise básica, ou seja: teor de argila, $\mathrm{pH}$, acidez potencial, índice SMP, fósforo $(\mathrm{P})$, potássio $(\mathrm{K})$, matéria orgânica $(\mathrm{MO})$, cálcio $(\mathrm{Ca})$, magnésio $(\mathrm{Mg})$, alumínio trocável $\left(\mathrm{Al}^{+3}\right)$, capacidade de troca de cátions (CTC), saturação por $\mathrm{Al}(\mathrm{m} \%)$ e saturação das bases (V), de acordo com Oleynik et al. (1995).

\section{ANÁLISE DE DADOS}

A aplicação de métodos quantitativos em estudos ambientais é muito antiga e o seu objetivo é analisar os dados multidimensionais, com múltiplas variáveis para um mesmo ponto amostral, conseguindo a máxima interpretação contida nesses. Dessa forma, a análise 
multivariada se faz necessária para considerar a variabilidade de diferentes unidades de medidas e reduzir a dimensão dos dados, ciente de que toda a análise estatística prescinde da organização, padronização e normalização das informações.

Para os dados analíticos das campanhas de monitoramento, disponíveis para esses pontos, foi realizada uma análise de componentes principais (ACP). Por essa ferramenta de estatística multivariada, buscou-se entender o relacionamento entre as variáveis elencadas para o estudo para cada um dos pontos, a similaridade de dados de diferentes campanhas de monitoramento e quais os componentes que as determinam (LANDIM, 2011).

A ACP é uma técnica estatística multivariada que transforma um conjunto de variáveis originais em um conjunto reclassificado, de mesma dimensão, denominado de componentes principais. De acordo com Vicini (2008), cada componente formado é uma combinação linear da soma de variáveis originais, interdependentes entre si, com o propósito de reter o máximo de informação, em termos de variação total contida nos dados. No software $M V S P^{\circledR}$, foi possível aplicar a técnica de ACP e verificou-se qualidade de representação através de gráfico euclidiano bidimensional.

As análises foram realizadas a partir de dados de 6 diferentes áreas monitoradas durante 6 campanhas analíticas, para 12 diferentes parâmetros físico-químicos e de fertilidade dos solos construídos, em áreas que foram recuperadas ambientalmente. O estudo iniciou no ano de 2013, com coletas semestrais, estendendo-se até o ano de 2014, denominadas de campanhas 7, 8, 9 e 10. As campanhas 12 e 14 aconteceram nos anos de 2015 e 2016 com repetição anual. Esses locais constituíam passivos de mineração a céu aberto, foram recuperados fisicamente e estão em processo de acompanhamento da evolução das condições químicas e biológicas, inserindo-se o parâmetro solo.

\section{RESULTADOS E DISCUSSÕES}

Segundo Landim (2011), a análise estatística multivariada deve ser precedida da montagem de uma matriz de dados, com a padronização e a normalização dos dados brutos (Tabela 1). Dessa forma, houve a necessidade do pré-processamento dos dados analíticos, tornando-os aptos para a análise estatística. A etapa de tratamento foi subdividida na averiguação da normalidade da série de dados de cada variável, e na padronização deles. 
Tabela 1 - Condições edáficas de áreas de passivo ambiental ao longo do estudo

\begin{tabular}{|c|c|c|c|c|c|c|c|c|c|c|c|}
\hline ÁREA & CAMP. & $\begin{array}{c}\text { ARGILA } \\
(\%)\end{array}$ & $\begin{array}{c}\mathrm{Ph} \\
\text { (em } \\
\text { água) }\end{array}$ & SMP & $\begin{array}{c}\mathbf{P} \\
\left(\mathbf{m g} / \mathrm{dm}^{3}\right)\end{array}$ & $\begin{array}{c}\mathrm{K} \\
\left(\mathrm{mg} / \mathrm{dm}^{3}\right)\end{array}$ & $\begin{array}{l}\text { MO } \\
(\%)\end{array}$ & SB & $\begin{array}{c}\text { CTC } \\
\left(\mathrm{cmolc} / \mathrm{dm}^{3}\right)\end{array}$ & V (\%) & $\begin{array}{c}m \\
(\%)\end{array}$ \\
\hline \multirow{6}{*}{ Locks } & 7 & 29 & 4,5 & 4,5 & 17,4 & 87 & 2,1 & 3,2 & 2,4 & 5,94 & 30,35 \\
\hline & 8 & 40 & 4,2 & 4,2 & 10,6 & 86 & 1,3 & 1,5 & 0,5 & 2,25 & 36,7 \\
\hline & 9 & 29 & 3,9 & 4,4 & 2,4 & 78 & 1,3 & 1,5 & 1,19 & 2,89 & 8,98 \\
\hline & 10 & 31 & 4,8 & 5,9 & 55,9 & 91 & 1,8 & 9,37 & 0,61 & 10,21 & 15,11 \\
\hline & 12 & 43 & 4,2 & 4,4 & 10 & 62 & 0,8 & 2,9 & 2,2 & 5,26 & 31,76 \\
\hline & 14 & 40 & 4,2 & 4,3 & 8,8 & 70 & 2,3 & 2,7 & 2,2 & 5,05 & 34,35 \\
\hline \multirow{6}{*}{ 3АЗB } & 7 & 38 & 4,3 & 4,3 & 19,2 & 88 & 1,7 & 3 & 2,3 & 5,63 & 30,04 \\
\hline & 8 & 40 & 4,5 & 4,4 & 10,7 & 86 & 0,9 & 1,6 & 0,7 & 2,55 & 29,93 \\
\hline & 9 & 31 & 4,9 & 5,5 & 23,2 & 130 & 2,3 & 4,36 & 2,51 & 7,20 & 8,52 \\
\hline & 10 & 29 & 4,7 & 5,7 & 9,3 & 99 & 1,2 & 8,35 & 0,87 & 9,47 & 15,67 \\
\hline & 12 & 39 & 4,7 & 4,9 & 10 & 2 & 0,9 & 2,6 & 1,9 & 4,51 & 19,62 \\
\hline & 14 & 40 & 5,2 & 5,2 & 9,9 & 60 & 2,5 & 3,8 & 2,5 & 6,41 & 17,51 \\
\hline \multirow{6}{*}{ Bett } & 7 & 35 & 4,8 & 4,8 & 21,3 & 109 & 0,8 & 5,2 & 4 & 9,59 & 26,89 \\
\hline & 8 & 40 & 4,7 & 4,6 & 5 & 88 & 0,6 & 1,8 & 0,8 & 2,85 & 24,61 \\
\hline & 9 & 40 & 4,3 & 4,6 & 3,3 & 92 & 0,9 & 2,72 & 1,88 & 4,83 & 26,64 \\
\hline & 10 & 24 & 5 & 5,5 & 2,3 & 72 & 0,7 & 8,71 & 0,55 & 9,44 & 15,87 \\
\hline & 12 & 50 & 4,6 & 4,8 & 16,5 & 16 & 1 & 4 & 3,6 & 7,64 & 24,54 \\
\hline & 14 & 47 & 5 & 5,1 & 3,8 & 60 & 1,6 & 4 & 2,8 & 6,92 & 19,02 \\
\hline \multirow{6}{*}{$\begin{array}{l}\text { Rio do } \\
\text { Meio }\end{array}$} & 7 & 36 & 4,5 & 4,2 & 8 & 85 & 0,4 & 0,9 & 0,6 & 1,83 & 36,28 \\
\hline & 8 & 35 & 4,7 & 5,1 & 7,1 & 204 & 1,1 & 2,6 & 0,7 & 16,11 & 7,85 \\
\hline & 9 & 31 & 4,6 & 4,7 & 4,3 & 81 & 1 & 2,46 & 1,7 & 4,37 & 7,22 \\
\hline & 10 & 62 & 5,7 & 6,8 & 13,8 & 93 & 1,4 & 9,5 & 1,63 & 11,37 & 11,37 \\
\hline & 12 & 36 & 5,7 & 6,4 & 6,5 & 78 & 0,8 & 4,9 & 4,7 & 9,76 & 12,56 \\
\hline & 14 & 31 & 4,6 & 5,2 & 4,7 & 88 & 2,1 & 3,4 & 2,3 & 5,97 & 16,77 \\
\hline \multirow{6}{*}{$\begin{array}{c}\text { Zona } \\
4\end{array}$} & 7 & 57 & 4,5 & 4,5 & 9,3 & 93 & 1,3 & 2,7 & 1,7 & 4,74 & 29,15 \\
\hline & 8 & 60 & 4,3 & 4,6 & 7,6 & 92 & 0,7 & 1,4 & 0,3 & 1,97 & 23,73 \\
\hline & 9 & 33 & 4,3 & 4,9 & 17,6 & 62 & 0,8 & 1,52 & 1,25 & 2,92 & 18,33 \\
\hline & 10 & 21 & 4,8 & 5,5 & 56,2 & 77 & 1,2 & 5,48 & 0,54 & 6,21 & 12,24 \\
\hline & 12 & 27 & 4,3 & 5 & 6,1 & 52 & 0,5 & 1,4 & 0,9 & 2,43 & 16,03 \\
\hline & 14 & 32 & 4,9 & 6,2 & 10,9 & 64 & 1,4 & 1,6 & 1 & 2,76 & 6,23 \\
\hline \multirow{6}{*}{$\begin{array}{l}\text { Coréia } \\
\text { II }\end{array}$} & 7 & 40 & 4,1 & 4,1 & 50 & 138 & 2,6 & 2,3 & 1,4 & 4,16 & 42,81 \\
\hline & 8 & 42 & 4,3 & 4,3 & 17 & 142 & 2,3 & 1,6 & 0,6 & 2,56 & 33,31 \\
\hline & 9 & 36 & 4,2 & 4,3 & 7,8 & 98 & 2,5 & 1,37 & 0,91 & 2,53 & 33,23 \\
\hline & 10 & 33 & 4,8 & 5 & 56,7 & 10 & 3,6 & 7,97 & 0,49 & 8,49 & 22,19 \\
\hline & 12 & 50 & 4,6 & 4,5 & 11,4 & 96 & 2,5 & 2,5 & 1,4 & 4,15 & 28,55 \\
\hline & 14 & 44 & 4,3 & 4,5 & 11,3 & 262 & 2,7 & 1,8 & 0,9 & 3,36 & 26,36 \\
\hline
\end{tabular}

$\mathrm{P}$ - fósforo; $\mathrm{K}$ - potássio; MO - matéria orgânica; $\mathrm{SB}$ - soma de bases; CTC - capacidade de troca de cátions; V - saturação por bases; $\mathrm{m}$ - saturação por alumínio.

Fonte: Elaborada pelos autores.

Após a padronização e normalização, os dados foram importados para o software MVSP ${ }^{\circledR}$. Nesse programa, efetuou-se a ACP obtendo-se uma matriz de correlação múltipla das variáveis (Tabela 2), além da determinação do peso dos eixos dos componentes principais e da possibilidade de se averiguar os pesos das variáveis conforme distintos eixos.

Tabela 2 - Matriz de similaridade entre os parâmetros da pesquisa. Os destaques refletem as maiores correlações entre os parâmetros

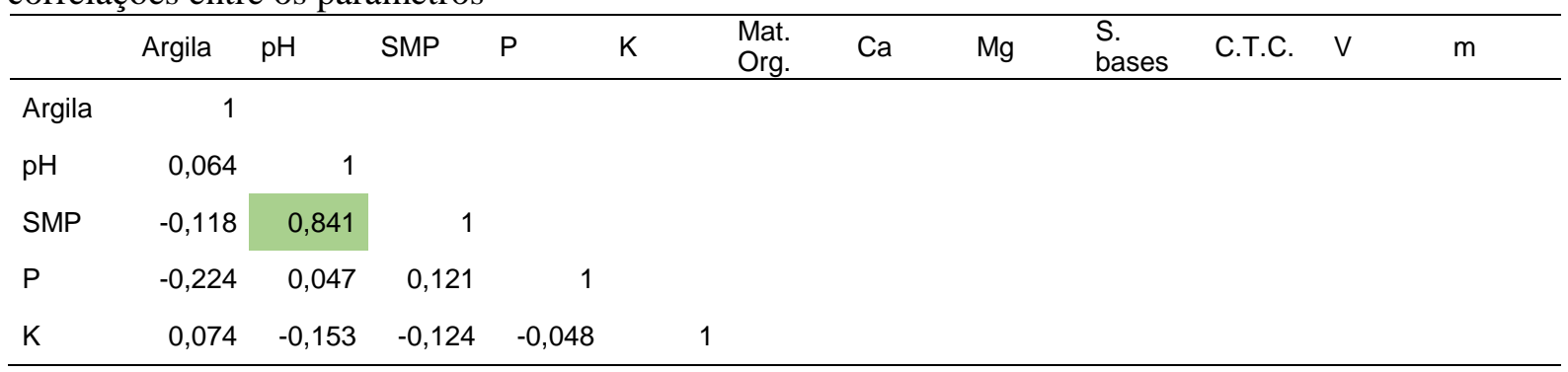




\begin{tabular}{|c|c|c|c|c|c|c|c|c|c|c|c|c|}
\hline $\begin{array}{l}\text { Mat. } \\
\text { Org. }\end{array}$ & 0,028 & $-0,061$ & $-0,11$ & 0,427 & 0,216 & 1 & & & & & & \\
\hline $\mathrm{Ca}$ & $-0,125$ & 0,629 & 0,68 & 0,421 & $-0,161$ & 0,123 & 1 & & & & & \\
\hline $\mathrm{Mg}$ & 0,119 & 0,368 & 0,176 & $-0,168$ & $-0,195$ & $-0,047$ & 0,086 & 1 & & & & \\
\hline $\begin{array}{l}\mathrm{S} \text {. } \\
\text { bases }\end{array}$ & $-0,087$ & 0,611 & 0,589 & 0,204 & 0,122 & 0,029 & 0,731 & 0,296 & 1 & & & \\
\hline C.T.C. & 0,329 & $-0,535$ & $-0,748$ & 0,067 & 0,079 & 0,201 & $-0,367$ & $-0,053$ & $-0,445$ & 1 & & \\
\hline V & $-0,114$ & 0,848 & 0,936 & 0,233 & $-0,135$ & $-0,009$ & 0,854 & 0,301 & 0,712 & $-0,604$ & 1 & \\
\hline \multirow[t]{2}{*}{$\mathrm{m}$} & $-0,058$ & $-0,711$ & $-0,647$ & $-0,141$ & 0,271 & $-0,03$ & $-0,597$ & $-0,722$ & $-0,58$ & 0,405 & $-0,76$ & \\
\hline & Argila & & SMP & $P$ & $\mathrm{~K}$ & $\begin{array}{l}\text { Mat. } \\
\text { Org. }\end{array}$ & $\mathrm{Ca}$ & $\mathrm{Mg}$ & $\begin{array}{l}\mathrm{S} . \\
\text { bases }\end{array}$ & C.T.C. & V & $\mathrm{m}$ \\
\hline
\end{tabular}

Fonte: Os autores.

Ressalta-se que estes dados foram inicialmente tratados, com todas as variáveis e casos dispostos na Tabela 1 e com esta técnica foi possível gerar gráfico euclidiano bidimensional (Figura 2) que cruza os eixos 1 e 2. Desta forma, as possíveis relações entre variáveis e casos puderam ser percebidas.

Figura 2 - Diagrama bidimensional executado a partir do cruzamento dos eixos 1 E 2 obtidos

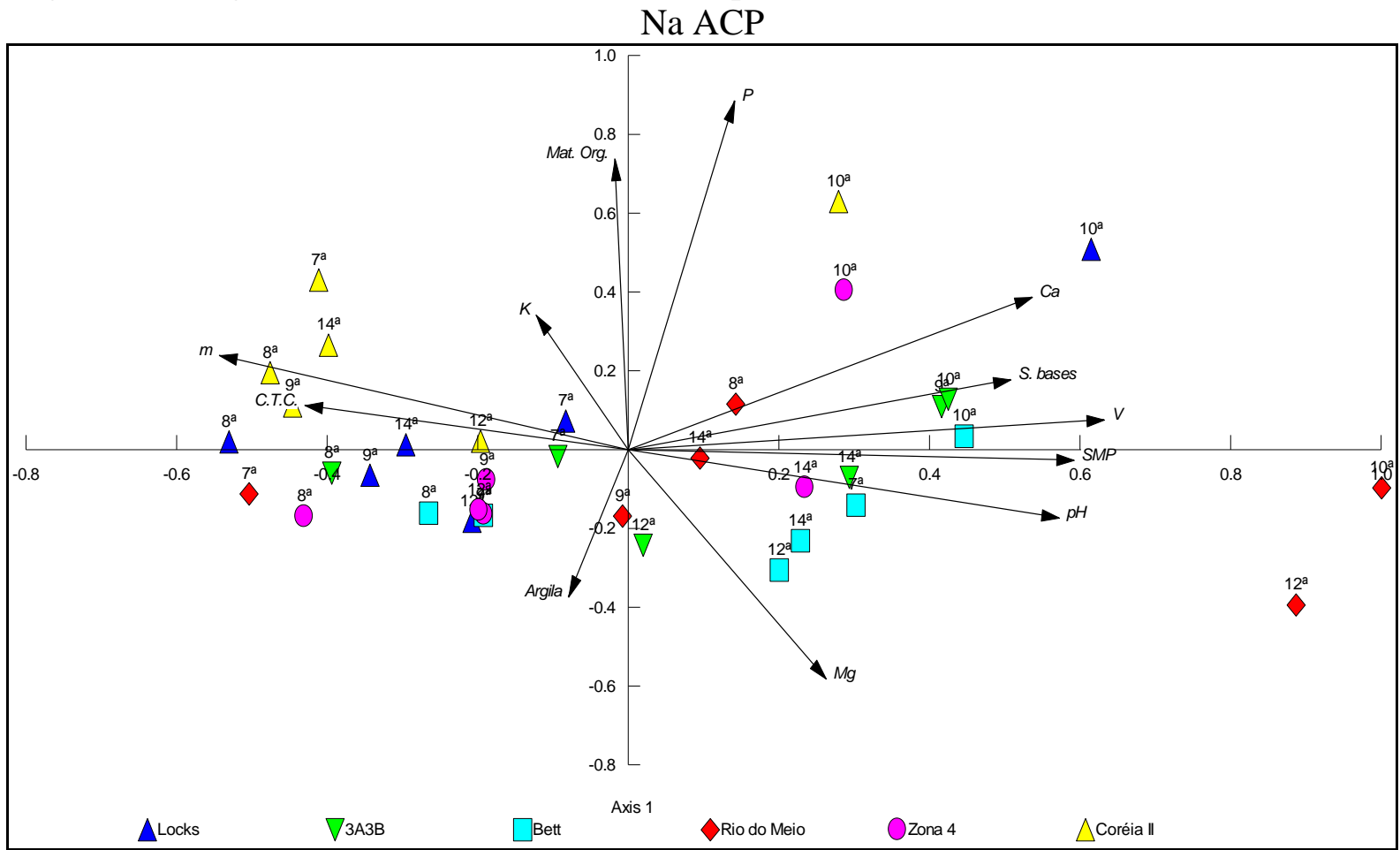

Fonte: Elaborada pelo autor.

A relação entre os casos (áreas de estudo, rotuladas por campanhas amostrais) e os vetores (parâmetros qualitativos do estudo) denota que, quando os casos (áreas de estudo) estão posicionados no lado direito (em relação ao eixo vertical) do gráfico, os mesmos são influenciados, principalmente, por vetores de soma de bases, $\mathrm{pH}$, saturação por bases e cálcio, 
ou seja, ambientes mais favoráveis ao desenvolvimento de vegetação. Por outro lado, os casos dispostos à esquerda do gráfico, sob influência principal da saturação por alumínio (m\%), indicam um solo distrófico, com baixas condições edáficas. Dessa forma, essa espacialização dos casos e suas campanhas, determinados pelos vetores descritores dos parâmetros físico/químicos, contribui para o acompanhamento da evolução do estágio de recuperação dos locais analisados, bem como comparação entre as áreas.

A análise do diagrama bidimensional (Figura 2), proveniente da ACP, permite verificar, em relação aos parâmetros e seu relacionamento, a oposição entre m, CTC, fortemente correlacionados positivamente, em relação ao pH, SMP, V, S. bases e Ca. Num componente secundário, matéria orgânica e P relacionam-se positivamente, em contraposição à variável argila. Essa interpretação dos vetores das variáveis, para todos os parâmetros, define, por exemplo, que solos ricos em argila possuem pouca MO, por exemplo. Já a distribuição dos casos no gráfico, ou seja, de todas as campanhas individualizadas para cada área de estudo, conforme feição geométrica específica, define que a variância principal entre os parâmetros ocorre ao longo do eixo das abcissas, ou seja, ao longo do componente principal 1, dispersando-se os casos da esquerda para a direita da figura.

Ao se analisar o comportamento do solo ao longo do monitoramento, é possível observar uma evolução abrupta da qualidade na $10^{\circ}$ campanha, com um significativo retrocesso na $14^{\circ}$ campanha, em níveis muito próximos ao que vinha sendo encontrado ao longo do período. Considerando a normalidade do processo de recuperação e, que nessa campanha as análises foram realizadas por outro laboratório, sugere-se que possa ter ocorrido um erro laboratorial e, por esse motivo, não se considera essa campanha como um resultado confiável.

Os altos teores de argila, com concentrações acima de 40\%, são sinônimos de baixa qualidade edáfica, visto que a concentração excessiva dessa pode determinar condições físicas de compactação, impossibilitando o desenvolvimento de raízes, bem como a sustentabilidade deste solo.

A saturação por alumínio tem ação muito forte sobre as condições da qualidade do solo, sendo que o pH, o índice SMP, o Ca e o Mg são fortemente influenciados negativamente por este fator. Da mesma forma, quando se reduzem os valores da saturação por alumínio, ocorre o aumento da saturação por bases, elevando a disponibilidade de macronutrientes e do $\mathrm{pH}$, melhorando as condições de desenvolvimento vegetal do solo.

Pela posição no gráfico, as áreas Coreia e Locks apresentam-se com os solos de pior qualidade, enquanto que nas áreas Bett e 3A3B se encontram os solos com melhor qualidade. 
As áreas Rio do Meio e Zona 4 apresentaram grandes variações entre as campanhas, demonstrando uma instabilidade, porém com perspectivas de evolução.

É importante destacar que, em virtude da carência de parâmetros específicos para solos de mineração, os resultados de trabalhos que levem em consideração parâmetros de fertilidade de solos influenciados pela mineração, mesmo que parâmetros vinculados a áreas agrícolas, são de extrema importância. Deve-se ressaltar ainda que, para que um solo seja considerado fértil, além de conter quantidades suficientes de nutrientes essenciais às plantas, deve estar livre de substâncias tóxicas e apresentar propriedades físicas satisfatórias (SILVA et al., 2004).

\section{CONCLUSÕES}

$\mathrm{Na}$ avaliação temporal das áreas monitoradas, evidenciou-se uma evolução positiva ao longo das 6 campanhas de monitoramento na maioria das áreas. As áreas Locks e Coreia II, ao contrário das demais, se apresentam com um desenvolvimento negativo, sendo evidente que as áreas não atingiram o objetivo da recuperação ambiental realizada. Já as áreas Bett e 3A3B destacam-se com resultados positivos, com tendência a atingir um nível ideal de sustentabilidade do solo.

Em condições de campo, é possível observar, em todas as áreas estudadas, a influência que a saturação por alumínio ( $\mathrm{m} \%)$ exerce sobre diversas variáveis importantes na qualidade nutricional do solo, evidenciando que, quanto maior a saturação por alumínio (\%), menores são os valores de $\mathrm{pH}$, do índice SMP, de magnésio, de cálcio e de saturação por bases do solo, caracterizando-o, sob essas condições, como de baixa fertilidade.

A concentração de argila e de matéria orgânica no solo influenciam diretamente sobre as condições de desenvolvimento radicular da vegetação, sendo que, um solo que apresente excesso de argila associado a baixas concentrações de matéria orgânica, oferece condições de compactação e perda de solo por erosão, gerando limitações ao desenvolvimento da vegetação na área.

Diante do exposto, salienta-se que os índices de concentração dos atributos do solo, aqui utilizados para análise da fertilidade, são baseados em critérios agronômicos padronizados para solos agrícolas e não para áreas influenciadas pela mineração de carvão, ou seja, a adoção destes critérios deve ser efetuada com cautela quando da análise de solos construídos. Porém, estudos que avaliem a evolução das condições edáficas de um solo construído são de extrema importância, buscando identificar os melhores métodos de recuperação das áreas degradadas. Deve-se considerar, ainda, que a disponibilidade de 
nutrientes e a velocidade de decomposição da matéria orgânica são influenciadas tanto pela amplitude térmica quanto pela evapotranspiração do solo, umidade relativa e intensidade luminosa, as quais variam sazonalmente na latitude em que foi realizado este estudo.

\section{REFERÊNCIAS}

ARAÚJO, F. S.; MARTINS, S. V.; MEIRA NETO, J. A. A.; LANI, J. L.; PIRES, I. E. Florística da vegetação arbustivo-arbórea colonizadora de uma área degradada por mineração de caulim, em Brás Pires, MG. Revista Árvore, Viçosa, MG. v. 29, n. 6, p. 983-992, 2005. BELOLLI, M.; QUADROS, J.; GUIDI, A.; História do Carvão em Santa Catarina. Criciúma/SC. Imprensa oficial do estado de Santa Catarina, 2002.

BRASIL. Ministério da Agricultura. Departamento Nacional da Produção Mineral (DNPM). Perfil analítico do carvão. 2. ed. Porto Alegre, 1987 (Boletim, 6).

CAMPOS, M. L.; ALMEIDA, J. A.; SOUZA, L. S. Avaliação de três áreas de solo construído após mineração de carvão a céu aberto em Lauro Müller, Santa Catarina. Revista Brasileira de Ciência do Solo, Viçosa, MG, v.27, p. 1123-1137, 2003. CIRAM - CENTRO DE INFORMAÇÕES DE RECURSOS AMBIENTAIS E DE HIDROMETEOROLOGIA DE SANTA CATARINA/EPAGRI - EMPRESA DE PESQUISA AGROPECUÁRIA E DE EXTENSÃO RURAL DE SANTA CATARINA. Estações meteorológicas do município de Criciúma: monitoramento de dados meteorológicos para prevenção de enchentes e deslizamentos. Disponível em: <http://ciram.epagri.sc.gov.br/agroconnect/>. Acesso em: 2 fev. 2017. COMISSÃO DE QUÍMICA E FERTILIDADE DO SOLO-RS/SC. Manual de Calagem e Adubação para os Estados do Rio Grande do Sul e de Santa Catarina. 11. ed. Porto Alegre: SBCS-Núcleo Regional Sul/UFRGS, 2016. 376p.

CONAMA - Conselho Nacional do Meio Ambiente. Resolução número 001/86. Ministério do Meio Ambiente.

EMBRAPA. Sistema brasileiro de classificação de solos. Centro Nacional de Pesquisa de Solos. 2. ed. Brasília, 2006.

FANNING, D.S.; FANNING, M.C.B. Soil: morphology, genesis and classification. New York: J. Willey, 1989.

FUNGARO, A. D.; IZIDORO, C. de J. Remediação de drenagem ácida de mina usando zeólitas sintetizadas a partir de cinzas leves de carvão. Química Nova, v. 29, p. 735-740. 2006.

LANDIM, P. M. B. Análise estatística de dados geológicos multivariados. São Paulo: Oficina de Textos, 2011.

LEITE, P. F.; KLEIN, R. M. Vegetação. In: Instituto Brasileiro de Geografia e Estatística Geografia do Brasil - IBGE. Geografia do Brasil: Região Sul. Rio de Janeiro: 1990. v.2. p.113-150.

OLEYNIK, J.; BRAGAGNOLO, N.; BUBLITZ, U.; SILVA, J. C. C. Análises de solo: tabelas para transformação de resultados analíticos e interpretação de resultados. Curitiba: EMATER - Paraná, 1995 (Informação Técnica, 31).

ALVES, M. C.; SOUZA, Z. M. recuperação de área degradada por construção de hidroelétrica com adubação verde e corretivo. R. Bras. Ci. Solo, v.32, p.2505-2516, 2008. PEEL, M. C., FINLAYSON, B. L., McMAHON, T. A. Updated world map of the KöppenGeiger climate classification, Hydrology and Earth System. Sciences, v.11, p. 1633-1644, 2007.

PINTO, L. F. S. Potencial de acidificação e de neutralização dos materiais geológicos para a composição do solo construído em áreas de mineração de carvão. 1997. 186 f. 
Tese (Doutorado em Ciência do Solo) - Universidade Federal do Rio Grande do Sul, Porto Alegre, 1997.

RODRIGUES, L. A.; CARVALHO, D. A.; OLIVEIRA FILHO, A. T.; CURI, N. Efeitos de solos e topografia sobre a distribuição de espécies arbóreas em um fragmento de floresta Estacional Semidecidual em Luminárias, MG. Revista Árvore, Viçosa, MG. v. 33, n. 1, p. 25-35, 2007.

SILVA, S. R.; PROCOPIO, S. O.; QUEIROZ, T. F. N.; DIAS, L. E. Caracterização de rejeito de mineração de ouro para avaliação de solubilização de metais pesados e arsênio e revegetação local. Revista Brasileira de Ciência do Solo, Viçosa, MG, v. 28, n. 1, p. 189196, 2004.

TORREZANI, N. C.; OLIVEIRA, E. F. Problemas ambientais decorrentes da exploração do carvão mineral e a aplicação da ecotoxicologia aquática como ferramenta de biomonitoramento. Oecologia Australis, Londrina, PR, v. 17, n. 4, p. 509-521, dez. 2013. VICINI, L. Análise multivariada da teoria à prática. Santa Maria: UFSM, CCNE, 2005. 215 p. 